\title{
Reducing the Risk of Hospitalization for Nursing Home Residents: Effects and Facility
}

\section{Variation From OPTIMISTIC}

Justin Blackburn, Timothy E. Stump, Jennifer L. Carnahan, Susan E. Hickman, Wanzhu Tu, Nicole R. Fowler,

Kathleen T. Unroe

This is the author's manuscript of the article published in final edited form as:

Blackburn, J., Stump, T. E., Carnahan, J. L., Hickman, S. E., Tu, W., Fowler, N. R., \& Unroe, K. T. (2020). Reducing the Risk of Hospitalization for Nursing Home Residents: Effects and Facility Variation From OPTIMISTIC. Journal of the American Medical Directors Association, 21(4), 545-549.e1. https://doi.org/10.1016/j.jamda.2020.02.002 


\begin{abstract}
Objectives: The Optimizing Patient Transfers, Impacting Medical Quality, and Improving Symptoms: Transforming Institutional Care (OPTIMISTIC) project led to significant decreases in potentially avoidable hospitalizations of long-stay nursing facility residents in external evaluation. The purpose of this study was to quantify hospitalization risk from the start of the project and describe the heterogeneity of the enrolled facilities in order to better understand the context for successful implementation.
\end{abstract}

Design: Pre-post analysis design of a prospective intervention within a single group. Setting and Participants: 4,320 residents in the 19 facilities were included from admission until time to the first hospitalization.

Measures: Data were extracted from Minimum Data Set assessments and linked with facility-level covariates from the LTCFocus.org data set. Kaplan-Meier and Cox proportional hazards regression were used to assess risk of hospitalization during the pre-intervention period (2011-2012), a "rampup" period (2013-2014), and intervention period (2015-2016).

Results: The cohort consisted of 4,230 long-stay nursing facility residents. Compared to the preintervention period, residents during the intervention period had an increased probability of having no hospitalizations within 1 year, increasing from 0.51 to 0.57 , which was statistically significant $(\mathrm{p}<0.001)$. In adjusted Cox models, the risk of hospitalization was lower in the ramp-up period compared to the pre-period (Hazard ratio [HR] 0.85, 95\% confidence interval [CI] 0.75-0.95) and decreased further during the intervention period (HR 0.74, 95\% CI $0.65-0.84$ ).

Conclusions and Implications: As part of a large multi-site demonstration project, OPTIMISTIC has successfully reduced hospitalizations. However, this study highlights the magnitude and extent to which results differ across facilities. Implementing the OPTIMISTIC 
24 program was associated with a $16 \%$ risk reduction after the first 18 months and continued to a

25 final risk reduction of $26 \%$ after 5 and a half years. Although this model of care reduces

26 hospitalizations overall, facility variation should be expected.

27

28

29 


\section{Introduction}

Long-term nursing facility residents (hereafter called residents) are at high risk of

32 experiencing potentially avoidable hospitalizations. ${ }^{1-6}$ As many as $60 \%$ of resident

33 hospitalizations may be potentially avoidable, especially those related to five conditions:

34 pneumonia, congestive heart failure, urinary tract infections, dehydration, and chronic

35 obstructive pulmonary disease/asthma. ${ }^{2}$ Additionally, the likelihood of having a potentially avoidable hospitalization is associated with resident sociodemographics, facility characteristics

37 and substandard care practices, such as the type and level of staffing, their access to ancillary

38 services, and use of hospice..$^{1,7-9}$

In response to the high number of potentially avoidable hospitalizations of residents, the

40 Centers for Medicare and Medicaid Services (CMS) Innovations Center funded seven projects

41 under the National Initiative to Reduce Avoidable Hospitalizations Among Nursing Facility

42 Residents. Researchers and clinicians at [institution redacted] developed the Optimizing Patient

43 Transfers, Impacting Medical Quality, and Improving Symptoms: Transforming Institutional

44 Care (OPTIMISTIC) Model, which was one of the seven sites funded under this national clinical

45 demonstration project. ${ }^{10}$ In order to participate in the project, CMS required facilities to complete

46 a readiness review process as well as meet inclusion criteria which included having a minimum

47 3-star quality rating on the CMS Nursing Home Compare. In total, OPTIMISTIC recruited 19

48 facilities in Central Indiana to participate.

In brief, the OPTIMISTIC program employs full-time registered nurses to work with staff

50 at each facility to assess and address changes in resident condition and quality improvement

51 efforts. Additionally, OPTIMISTIC nurse practitioners provide in-person evaluations and

52 management of residents with acute condition changes. Evidence-based processes implemented 
53 under OPTIMISTIC include coordination of care through collaborative care reviews, Respecting

54 Choices Advanced Steps advance care planning facilitation, and the use of tools from

55 Interventions to Reduce Acute Care Transfers. ${ }^{3,11,12}$

56 The CMS evaluation of the national demonstration project found an overall reduction in

57 resident hospitalizations without increasing mortality across all participating sites. ${ }^{13,14}$ However,

58 variation was noted and OPTIMISTIC had a larger reduction in potentially avoidable

59 hospitalizations relative to other sites. ${ }^{10,14}$ The goal of these analyses was to further explore the

60 initial findings and quantify the timing and facility-level variation in the reduction of

61 hospitalization risk among facilities participating in OPTIMISTIC.

62

63 Methods

Full details of the OPTIMISTIC program, including facility and resident eligibility

65 criteria are described elsewhere. ${ }^{10}$ Nineteen facilities in Indiana were enrolled in the

66 OPTIMISTIC program in October 2012. Per CMS, the focus of OPTIMISTIC was long-stay

67 residents ( $>100$ days in a facility) or those with no documented discharge plans on the Minimum

68 Data Set (MDS). This analysis focused on the time-to-first hospitalization among eligible

69 residents to quantify the risk of hospitalization during OPTIMISTIC implementation. For these

70 analyses, eligible residents and their time-to-first hospitalization were identified from the MDS

71 and divided into three 18-month observation periods: pre-OPTIMISTIC (January 1, 2011 - June

72 30, 2012); OPTIMISTIC ramp-up (January 1, 2013 - June 30, 2014); and full OPTIMISTIC

73 intervention (January 1, 2015 - June 30, 2016). These observation periods corresponded to the

74 time before any OPTIMISTIC elements were implemented, when OPTIMISTIC staff were

75 present in facilities but their novel roles were still being defined and integrated into care 
practices, and when the full OPTIMISTIC model was in place. A second phase of the CMS

77 national demonstration project began in October 2016 for all seven sites, including

OPTIMISTIC, wherein additional facilities were enrolled. Consequently, 18-month observation

79 periods were selected to ensure homogenous non-overlapping intervals anchored by the period of full implementation of OPTIMISTIC from January 1, 2015 through June 30, 2016. Facility-level

81 covariate information was obtained from LTCFocus.org data including percentage of residents

82 with primary payer of Medicare, facility type, and average direct care hours per patient provided

83 by registered nurses, licensed practical nurses, certified nursing assistants, percentage female,

84 percentage white, average activities of daily living score, total beds, percentage with severe

85 cognitive impairment (as measured by the Cognitive Function Scale), and average Acuity Index

86 of the facility. Kaplan-Meier curves estimating the probability of a resident being

87 hospitalization-free from time of eligibility were calculated overall and separately for each

88 facility. Resident observations were censored if they left the facility, died, or had not been

89 hospitalized by the end of observation period. A log-rank test was used to compare the hospital-

90 free time distribution among the three time periods. Mixed-effects Cox proportional hazards

91 regression was used to estimate hazard ratios (HRs) and associated 95\% confidence intervals

92 (CIs) of hospitalization risk, adjusted for covariates; a random intercept was included in the

93 model to accounting for correlations among residents residing the same facility. Statistical

94 significance was assumed $\alpha=.05$. Analyses were performed using R software (R Foundation for

95 Statistical Computing, Vienna, Austria). The OPTIMISTIC demonstration project is approved by the [institution redacted] Institutional Review Board.

\section{$98 \quad$ Results}


The distribution of eligible residents $(\mathrm{N}=4,320)$ remained constant over time and facility,

100

101

102

103

104

105

106

107

108

109

110

111

112

113

114

115

116

117

118

119

120

121

with $n=1,434$ in the pre-intervention period, 1,426 in the ramp-up period, and 1,460 in the intervention period. Overall, facility \#18 had the largest proportion of residents with $8.1 \%$, whereas facility \#14 had the smallest with 2.5\%. Kaplan-Meier curves are shown for the overall sample (Figure 1). A statistically significant difference in time-to-hospitalization was observed across all three time periods (log-rank $\mathrm{p}<0.001)$; pairwise comparisons further revealed statistically significant differences across each time period (pre-intervention vs. ramp-up $\mathrm{p}=0.013$; pre-intervention vs. intervention $\mathrm{p}<0.001)$. The estimated probability of remaining hospitalization-free at 12 months was 0.51 during the pre-intervention, 0.57 during ramp-up, and 0.59 during the intervention. Kaplan-Meier estimates by facility (Figure 2) revealed that six of the nineteen facilities (32\%) exhibited statistically significant period effects indicating the risk of hospitalization differed over time for these facilities. Two facilities demonstrated a large decreased risk of hospitalization during the intervention (facilities \#6 and \#8). Another facility demonstrated an increased risk of hospitalization during the intervention (facility \#3). The other facilities showed a moderately lower risk of hospitalization during the intervention period (facilities \#2, \#10, and \#12). Additionally, although log-rank tests were not statistically significant at conventional levels, the survival curves for an additional 6 facilities displayed separation indicating lower hospitalization risk in the full OPTIMISTIC intervention period compared to other periods (facilities \#4, \#7, \#14,\#16,\#17, and \#18). The Cox mixed effects proportional hazards model (Table 1) showed that compared to the pre-intervention period, residents in the ramp-up period had a lower risk of hospitalization (HR 0.84, 95\% CI 0.750.95 ) and in the intervention period (HR $0.74,95 \%$ CI $0.65-0.84)$. Facility-level variance in unadjusted models was 0.09 and was reduced to approximately 0.01 with the addition of the 
122 facility-level baseline covariates. Inclusion of covariates did not change estimates for

123 intervention period effects.

\section{Discussion}

We estimated a $16 \%$ decrease in the risk of first all-cause hospitalization among residents

127 in OPTIMISTIC facilities after the first 18 months of the intervention; a risk that continued to 128 decline to a $26 \%$ reduction after 5 and a half years. There was notable heterogeneity in the effect 129 across facilities - although pooled estimates show OPTIMISTIC facilities collectively lowered 130 hospitalization risk over time, statistical differences across periods were not seen in all 131 participating facilities. Facility-level covariates decreased model variance and warrant further 132 investigation.

Facilities nationwide are changing the delivery of care to residents in response to state 134 and federal policies to incentivize value and quality, using elements similar to the OPTIMISTIC 135 clinical model. ${ }^{15}$ We observed reductions in hospitalization risk consistent with the overall 136 initiative's evaluation. ${ }^{14}$ Furthermore, our $26 \%$ risk reduction was consistent with another site's $13730 \%$ reduction. ${ }^{16}$ The overall initiative's qualitative findings hypothesized that heterogeneity in 138 the effects across states and facilities was attributable to variation in facility resource 139 commitment and implementation timelines. ${ }^{14}$ We provide evidence in support of this hypothesis, 140 such that timeliness and magnitude of effects differed by facility.

141 Linking specific characteristics of implementation, such as the facility's leadership 142 commitment and staff type and turnover, were beyond the scope of these analyses but could 143 potentially further explain the effect heterogeneity. However, some salient examples derived 144 from monthly calls with facilities and project operations notes warrant mention. Although the 
145 full OPTIMISTIC model was approved by all facilities, those with more engagement and 146 enthusiasm from the executive director, director of nursing, or parent corporation tended to

147 experience greater reductions in hospitalization risk. Such was the case in facilities \#8, \#6, \#10.

148 For example, the medical director of facility \#6 demonstrated this enthusiasm by actively

149 participating in OPTIMISTIC-led in-service activities, showing support and providing

150 perspective on how communication practices could be improved. In other cases, facilities were

151 not enthusiastic about integrating the OPTIMISTIC nurse into the existing staff. For example,

152 facility \#3 did not fully integrate the OPTIMISTIC nurse into the broader facility communication

153 channels, and often expressed "working in parallel" with the facility rather than in conjunction

154 with. A deeper understanding of successful implementation of programs like OPTIMISTIC

155 designed to reduce hospitalizations is still needed, but the Indiana experience highlights

156 engagement and enthusiasm from leadership accelerates change.

157 Our analyses did not estimate the direct effect attributable to the OPTIMISTIC

158 intervention, as there was no true comparison group. Rather, we sought to characterize

159 differences in hospitalization risk over time after implementation of OPTIMISTIC. Limiting to

160 the first hospitalization per resident may overestimate the effect on overall transfers if

161 subsequent transfers were numerous. In some facilities, the number of hospitalizations was low

162 and created instability in the facility-level Kaplan-Meier curves and could have resulted in a lack

163 of adequate statistical power for the log-rank test. Despite these limitations, our analyses enhance

164 the current understanding of hospitalization risk during implementation of a novel care model as

165 well describing differences in facility-level response.

\section{Conclusions and Implications}


168 Our results indicate that nursing facilities with varying characteristics can successfully adopt the 169 OPTIMISTIC model and sustainably improve the risk of hospitalizations within 18 months. Our 170 granular analyses are consistent with findings from the overall evaluation of the national

171 demonstration project, while revealing facility-level variation in the magnitude and timing of

172 effect. ${ }^{13,14}$ For facilities seeking to implement quality improvement efforts and/or new care 173 models aimed at reducing hospitalizations, the OPTIMISTIC model shows promise.

174

175 Conflicts of Interest

176 [Redacted author] is CEO and Founder of [redacted company name], a program to train nurses to 177 reduce nursing home hospital transfers. No other authors have potential conflicts of interest to 178 declare. 


\section{References}

1. Grabowski DC, Stewart KA, Broderick SM, Coots LA. Predictors of nursing home hospitalization: a review of the literature. Med Care Res Rev. 2008;65(1):3-39.

2. Ouslander JG, Lamb G, Perloe M, et al. Potentially avoidable hospitalizations of nursing home residents: frequency, causes, and costs: [see editorial comments by Drs. Jean F. Wyman and William R. Hazzard, pp 760-761]. J Am Geriatr Soc. 2010;58(4):627-635.

3. Ouslander JG, Naharci I, Engstrom G, et al. Root Cause Analyses of Transfers of Skilled Nursing Facility Patients to Acute Hospitals: Lessons Learned for Reducing Unnecessary Hospitalizations. J Am Med Dir Assoc. 2016;17(3):256-262.

4. Spector WD, Limcangco R, Williams C, Rhodes W, Hurd D. Potentially avoidable hospitalizations for elderly long-stay residents in nursing homes. Med Care. 2013;51(8):673-681.

5. Unroe KT, Hickman SE, Carnahan JL, Hass Z, Sachs G, Arling G. Investigating the Avoidability of Hospitalizations of Long Stay Nursing Home Residents: Opportunities for Improvement. Innov Aging. 2018;2(2):igy017.

6. Walsh EG, Wiener JM, Haber S, Bragg A, Freiman M, Ouslander JG. Potentially avoidable hospitalizations of dually eligible Medicare and Medicaid beneficiaries from nursing facility and Home- and Community-Based Services waiver programs. $J$ Am Geriatr Soc. 2012;60(5):821-829.

7. Intrator O, Zinn J, Mor V. Nursing home characteristics and potentially preventable hospitalizations of long-stay residents. J Am Geriatr Soc. 2004;52(10):1730-1736.

8. Ouslander JG, Berenson RA. Reducing unnecessary hospitalizations of nursing home residents. $N$ Engl J Med. 2011;365(13):1165-1167. 
202 9. Xing J, Mukamel DB, Temkin-Greener H. Hospitalizations of nursing home residents in the last year of life: nursing home characteristics and variation in potentially avoidable hospitalizations. J Am Geriatr Soc. 2013;61(11):1900-1908.

205 10. Unroe KT, Nazir A, Holtz LR, et al. The Optimizing Patient Transfers, Impacting Medical Quality, andImproving Symptoms:Transforming Institutional Care approach: preliminary data from the implementation of a Centers for Medicare and Medicaid

11. Ouslander JG, Perloe M, Givens JH, Kluge L, Rutland T, Lamb G. Reducing potentially 210 avoidable hospitalizations of nursing home residents: results of a pilot quality improvement project. J Am Med Dir Assoc. 2009;10(9):644-652.

212 12. Rask KJ, Hodge J, Kluge L. Impact of Contextual Factors on Interventions to Reduce Acute Care Transfers II Implementation and Hospital Readmission Rates. J Am Med Dir

215 13. Feng Z, Ingber MJ, Segelman M, et al. Nursing Facilities Can Reduce Avoidable Hospitalizations Without Increasing Mortality Risk For Residents. Health Aff (Millwood).

14. Ingber MJ, Feng Z, Khatutsky G, et al. Initiative To Reduce Avoidable Hospitalizations Among Nursing Facility Residents Shows Promising Results. Health Aff (Millwood). $2017 ; 36(3): 441-450$.

15. Daras LC, Wang JM, Ingber MJ, et al. What Are Nursing Facilities Doing to Reduce Potentially Avoidable Hospitalizations? J Am Med Dir Assoc. 2017;18(5):442-444. 
223 16. Rantz MJ, Popejoy L, Vogelsmeier A, et al. Successfully reducing hospitalizations of nursing home residents: results of the Missouri Quality Initiative. J Am Med Dir Assoc.

225 2017; 18(11):960-6.

226 
227 Figure 1 Kaplan-Meier curves for the total sample by time period $(\mathrm{N}=4,320)$

228 Figure 2 Kaplan-Meier curves for each OPTIMISTIC nursing home facility 


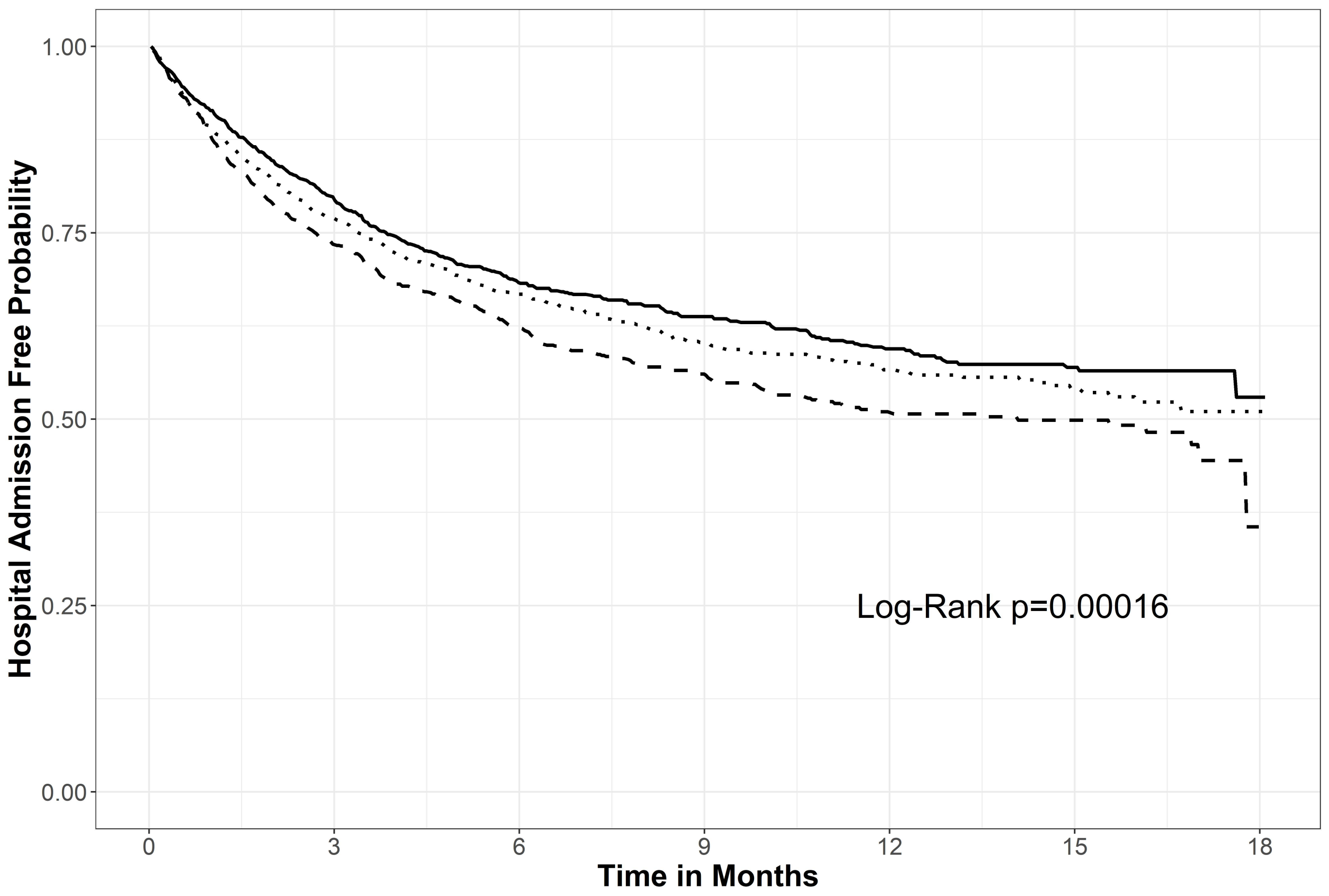

period $-{ }^{-}$01jan2011-30jun2012 $\cdots \cdots \quad$ 01jan2013-30jun2014 


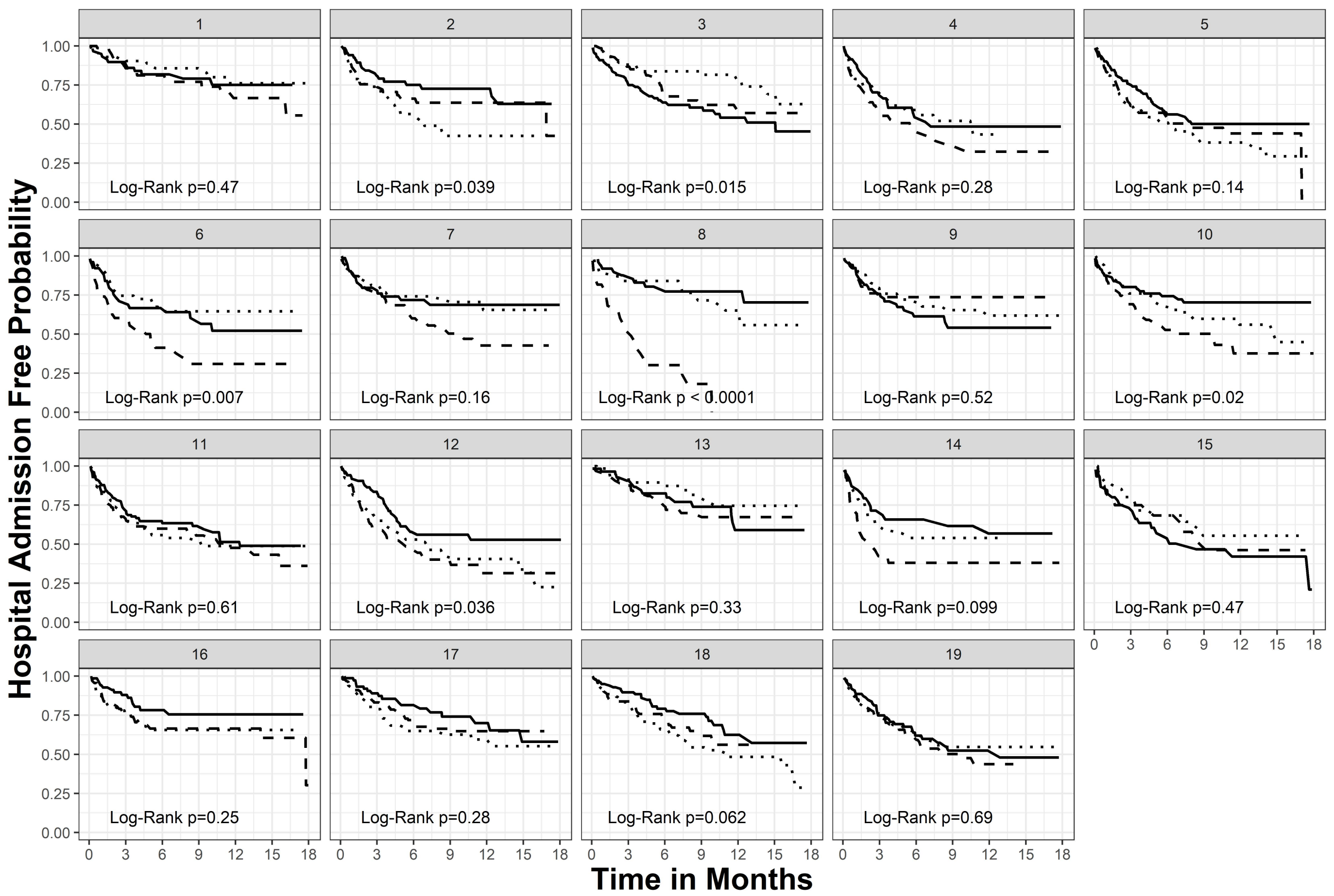

\section{Time in Months}


Table 1 . Frequency distribution and corresponding hazard ratios with $95 \%$ confidence intervals for each characteristic.

\begin{tabular}{|c|c|c|c|}
\hline & $\begin{array}{c}\text { Residents } \\
\mathrm{N}=4,320 \\
\mathrm{n}(\%) \text { or mean } \\
\text { (SD) }\end{array}$ & $\begin{array}{l}\text { Hazard } \\
\text { Ratio }\end{array}$ & $\begin{array}{l}95 \% \text { Confidence } \\
\text { Interval }\end{array}$ \\
\hline \multicolumn{4}{|l|}{ OPTIMISTIC Study Periods } \\
\hline $\begin{array}{l}\text { Pre-OPTIMISTIC Period } \\
\text { (January 1, } 2011 \text { - June 30, 2012) }\end{array}$ & $1,434(33.2 \%)$ & Reference & \\
\hline $\begin{array}{l}\text { OPTIMISTIC Ramp-up } \\
\text { (January 1, } 2013 \text { - June 30, 2014) }\end{array}$ & $1,426(33.0 \%)$ & 0.845 & $(0.748-0.954)$ \\
\hline $\begin{array}{l}\text { OPTIMISTIC Intervention } \\
\text { (January 1, } 2015 \text { - June } 30,2016 \text { ) }\end{array}$ & $1,460(33.8 \%)$ & 0.739 & $(0.653-0.836)$ \\
\hline \multicolumn{4}{|l|}{ Profit status of facilities } \\
\hline Not-for-profit & $3,200(74.1 \%)$ & Reference & \\
\hline For-profit facilities & $1,120(25.9 \%)$ & 1.212 & $(0.984-1.494)$ \\
\hline $\begin{array}{l}\text { Facility percentage of residents with } \\
\text { Medicare as primary payer }\end{array}$ & $19.2(7.4)$ & 1.023 & $(1.001-1.046)$ \\
\hline $\begin{array}{l}\text { Registered nurse hours per resident per day } \\
\text { (average) }\end{array}$ & $0.4(0.2)$ & 1.947 & $(1.403-2.700)$ \\
\hline $\begin{array}{l}\text { Licensed practical nurse hours per resident } \\
\text { per day (average) }\end{array}$ & $1.0(0.2)$ & 0.863 & $(0.552-1.349)$ \\
\hline $\begin{array}{l}\text { Certified nursing assistant hours per } \\
\text { resident per day (average) }\end{array}$ & $2.0(0.4)$ & 0.994 & $(0.722-1.369)$ \\
\hline Percentage female in facility & $63.8(7.2)$ & 0.993 & $(0.980,1.007)$ \\
\hline Percentage white in the facility & $77.2(20.5)$ & 0.998 & $(0.993,1.003)$ \\
\hline $\begin{array}{l}\text { Average activities of daily living score in } \\
\text { the facility }\end{array}$ & $18.1(1.5)$ & 0.996 & $(0.912,1.088)$ \\
\hline Total beds in the facility & $154.2(29.1)$ & 1.001 & $(0.998,1.004)$ \\
\hline $\begin{array}{l}\text { Average percentage of severe cognitive } \\
\text { impairment on the Cognitive Function Scale } \\
\text { in the facility }\end{array}$ & $6.9(7.4)$ & 1.001 & $(0.988,1.014)$ \\
\hline Average Acuity Index of the facility & $10.8(3.7)$ & 0.989 & $(0.973,1.007)$ \\
\hline
\end{tabular}

Note: hazard ratios estimated from multi-level Cox regression accounting for nesting of residents within facilities and adjusted for all other characteristics. Source: author's analysis of OPTIMISTIC data along with facility-level characteristics from LTCFocus (www.ltcfocus.org). 